\title{
ON THE MEASUREMENT OF INEQUALITIES IN HEALTH*
}

\author{
Adam Wagstaff, ${ }^{1}$ Pierella Pacl $^{2}$ and Eddy van Doorslaer ${ }^{3}$ \\ 'School of Social Sciences, University of Sussex, Brighton BNI 9QN, U.K. 'Department of Social \\ Sciences, City University, London ECIV OHB, U.K. and ' Institute of Medical Technology Assessment. \\ Erasmus University, 3000 DR Rotterdam, The Netherlands
}

\begin{abstract}
This paper offers a critical appraisal of the various methods employed to date to measure inequalities in health. It suggests that only two of these-the slope index of inequality and the concentration index-are likely to present an accurate picture of socioeconomic inequalities in health. The paper also presents several empirical examples to illustrate of the dangers of using other measures such as the range, the Lorenz curve and the index of dissimilarity.
\end{abstract}

Key words-inequalities in health, social class, comparative research, mortality, morbidity

\section{INTRODUCTION}

Much of the recent debate on inequalities of health in Britain and elsewhere dates back to the Black Report [1]. This concluded that, despite the setting up of the National Health Service in 1948, inequalities in mortality in England and Wales have been increasing ever since the 1930s. This conclusion has been challenged on a number of grounds, one being that the measure of inequality used-a variant of the range - was too crude and failed to take into account changes that will have tended to reduce inequality [2]. This led several researchers to investigate trends in inequalities in health using more sophisticated measures of inequality. However, the conclusions of these studies differ markedly: Le Grand et al. $[2,3]$ argue that inequalities in mortality have actually narrowed over the period in question, whilst other authors using different measures of inequality [4-6] argue the opposite.

It is surprising in view of both these radically different findings and the sheer amount of literature on inequalities in health that so little attention has been paid to the question of how health inequality is best measured. The primary objectives of this paper are, therefore, first to provide a critical review of the various measures of inequality that have been employed in the literature on inequalities in health to date and second to identify which measures are best suited to measuring health inequality. In Section 2 of the paper we identify six measures of inequality that have been employed in the literature to date, but argue that only two of these are really suited to the measurement of inequalities in health. The secondary purpose of the paper is to illustrate the usefulness of measures of inequality in comparative studies of health inequality. In Sections 3 and 4 we examine how measures of inequality can help in assessing trends in and cross-country differences in inequalities in morbidity (Section 3) and mortality (Section 4).

*This paper derives from the European Community's COMAC-HSR project on Equity in the Finance and Delivery of Health Care.

\section{MEASURING INEQUALITIES IN HEALTH}

The six measures of inequality that have been used to date in the literature on inequalities in health are: the range, the Gini coefficient (and the associated Lorenz curve), a pseudo-Gini coefficient (and an associated pseudo-Lorenz curve), the index of dissimilarity, the slope index of inequality (and the associated relative index of inequality) and the concentration index (and the associated concentration curve).

\section{The range}

This is the most frequently encountered measure of inequality in the literature on inequalities in health. Its use typically involves comparing the experiences of the top and bottom socioeconomic groups. Sometimes this comparison is presented in the form of the range itself: thus, for example, Lahelma and Valkonen [7] note that in Finland in 1964 there was a 17.5 percentage point difference between the top and bottom income groups in chronic illness rates. More often, however, the comparison is presented as the ratio of one extreme value to the other: thus the Black Report draws attention to the fact that in 1970-71 "men and women in occupational class V had a two-and-a-half times greater chance of dying before reaching retirement age than their professional counterparts in occupational class $I$ " $[1$, p. 51].

The defects of the range are obvious. First, it overlooks what is going on in the intermediate groups. The gap between the top and bottom groups might, for example, remain unchanged, but the extent of inequality between the intermediate groups might well be diminishing (or increasing). The authors of the Black Report were, in fact, well aware of this problem, noting, for example, that whilst the position of social class $\mathrm{V}$ improved between the 1959-63 and 1970-72 Decennial Supplements, the position of social class IV actually worsened [1, p. 67]. The second problem with the range is that it takes no account of the sizes of the groups being compared. This can lead to misleading results when comparisons are performed over time or across countries. For example, 
amongst males in England and Wales aged 15-64, social class I (professional) increased in size by $178 \%$ between 1931 and 1971, whilst social class V (unskilled) decreased by $35 \%[2$, p. 14]. The range measure overlooks this and therefore fails to take into account that the low death rates of the top social class now apply to a larger proportion of the population and that the high death rates of the bottom class apply to a smaller proportion. Clearly, the same problem arises in the context of cross-country comparisons if the various groups are not all the same size in each of the countries being compared.

\section{The Lorenz curve and Gini coefficient}

Various authors $[2-4,8-10]$ have suggested measuring inequalities in health using Lorenz curves and Gini coefficients. Le Grand et al. $[2,3,8,9]$ are, in fact, the only authors to date who have employed a true Lorenz curve. Their approach is illustrated in Fig. 1. The Lorenz curve-the curve labelled $g^{\text {hith }}(h)$-plots the cumulative proportions of the population (starting with the sickest person and ending with the healthiest) against the cumulative proportions of health. (The $h$ in brackets here is to emphasize that individuals are ranked by their health.) If health is equally distributed, the Lorenz curve coincides with the diagonal. Otherwise it lies beneath the diagonal.

The further the Lorenz curve is from the diagonal, the greater the degree of inequality. The area between the Lorenz curve and the diagonal (expressed as a proportion of the area beneath the diagonal) provides a measure of inequality. This measure, denoted below by $\mathrm{G}$, is the Gini coefficient. Since twice the area under the diagonal is equal to one half (the axes range from 0 to 1), the Gini coefficient is equal to twice the area between the Lorenz curve and the diagonal, or equivalently one minus the area under the Lorenz curve. It ranges from 0 (when there is complete equality and the Lorenz curve coincides with the diagonal) to 1 (when all the population's health is concentrated in the hands of one person and the Lorenz curve is -shaped).

The attractions of the Lorenz curve are clear: it reflects the experiences of all persons and not just those in, say, social classes I and V; moreover, because it does not involve stratifying the population

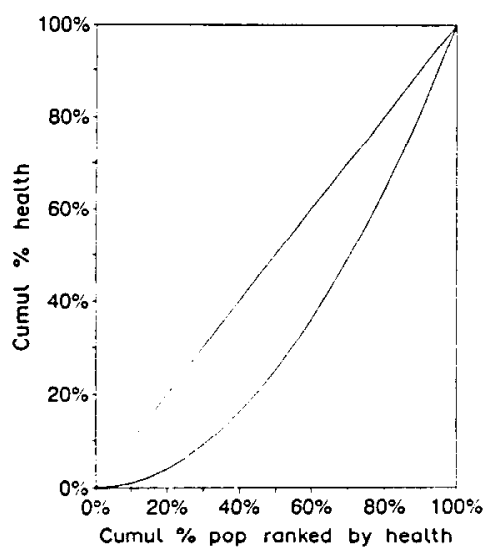

Fig. 1. Health Lorenz curve. by social class, it allows one to side-step all the problems associated with classifying people by social class, including the problem of changing class sizes. But the absence of a stratifying variable does mean that Le Grand and his colleagues end up addressing a different question from that being addressed in the bulk of the literature on inequalities in health, namely 'To what extent are there inequalities in health that are systematically related to socioeconomic status?' That the analysis fails to capture the socioeconomic dimension to inequalities in health is obvious. Any change in the distribution of health that keeps the mean level of health the same but involves a sick person getting healthier and a healthy person getting sicker is registered as a reduction in inequality in health irrespective of the socioeconomic status of the persons concerned. Inequality as measured by Le Grand and colleagues would still fall even if the sick person getting healthier were a member of social class I and the healthy person getting sicker were a member of social class $V$. Thus the fact that the Gini coefficient for age-at-death (Le Grand's measure of health) has fallen in England and Wales since 1941 is perfectly consistent with there having been increasing socioeconomic inequalities in age-at-death during this period.

Whether this insensitivity of the Lorenz curve to the socioeconomic dimension of inequalities in health is a defect depends clearly on the question one is seeking to answer. It clearly is a defect if one takes the view - as many do-that what is interesting - and indeed worrying - about inequalities in health is not that they exist, but that they mirror inequalities in socioeconomic status [11]

\section{Pseudo Lorenz curves}

At a first glance it would seem that Preston et al. [4] and Leclerc et al. [10] overcome this defect. Though they also claim to employ the Lorenz curve, their approach is in fact quite different from that of Le Grand and his colleagues. They employ grouped data rather than individual-level data. The groups, however, arc not health classes (as would have been expected given Fig. 1), but rather occupational classes. These classes are then ranked by their mortality, beginning with the class with the lowest mortality. The 'Lorenz' curve as used in Refs [4] and [10] thus graphs the cumulative percentage of the populationgrouped into occupational classes which are then ranked by health-against the cumulative percentage of deaths.

Because the population is assigned to groups according to their social class rather than health (even though the classes are then ordered by their health) means that the curve is not actually a Lorenz curve at all. Nonetheless, this pseudo-Lorenz curve-like the true Lorenz curve-still fails to reflect the socioeconomic dimension to inequalities in health, the reason being that classes are ordered according to their health. This means that the approach would be unable to differentiate between a situation where the sickest socioeconomic group of the population is made up of millionaires and one where the sickest socioeconomic group is made up of unskilled manual workers [2, p. 23].

One implication of this is that it will register positive inequality even when no class gradient exists. 


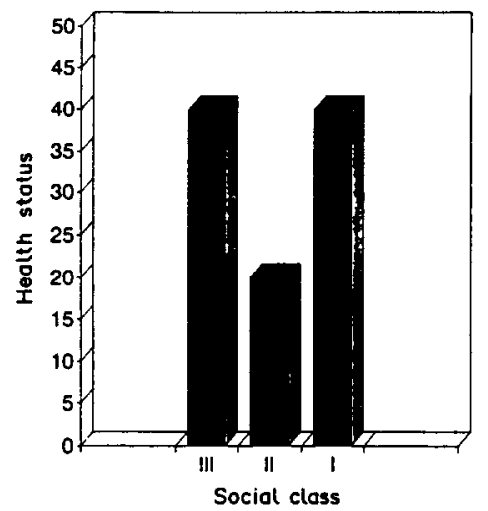

Fig. 2. Hypothetical distribution of health across social classes.

Consider Fig. 2. The three classes are identical in size. Classes I and III enjoy the same mean health status, but members of class II are less healthy. There is, however, no class gradient. Despite this, the pseudoLorenz curve lies beneath the diagonal (Fig. 3)classes are ordered as II, III and I-and the pseudo-Gini coefficient is positive (its value in this case is 0.133 ). The pseudo-Lorenz curve would also fail to detect a reversal in the class gradient of the type that occurred in mortality from heart disease in the 1950s [12]. To see this compare Figs 4 and 5. In Fig. 4 the class gradient favours the lowest class (as was the case with mortality from coronary heart disease up to the 1950s), whilst in Fig. 5 the gradient favours the highest class (as has been the case with mortality from coronary heart disease since the 1950s). Yet the pseudo-Lorenz curves and pseudoGini coefficients for the situations depicted in Figs 4 and 5 are identical. Indeed, they are identical to the Lorenz curve and Gini coefficient for the situation in Fig. 2.

\section{The index of dissimilarity}

Another index of inequality that has been used in the literature on inequalities in health $[4,6]$ is the index of dissimilarity. Suppose there are $j=1, \ldots, J$ socioeconomic groups. Then the index of dissimilarity (ID) is

$$
\mathrm{ID}=\frac{1}{2} \Sigma_{j}\left|s_{j h}-s_{j p}\right|,
$$

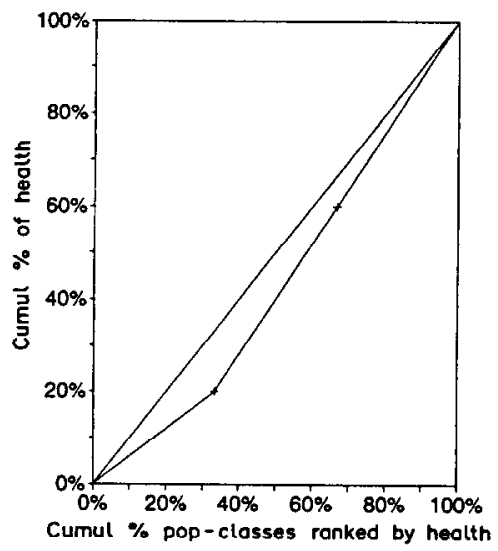

Fig. 3. Lorenz curve for Fig. 2.

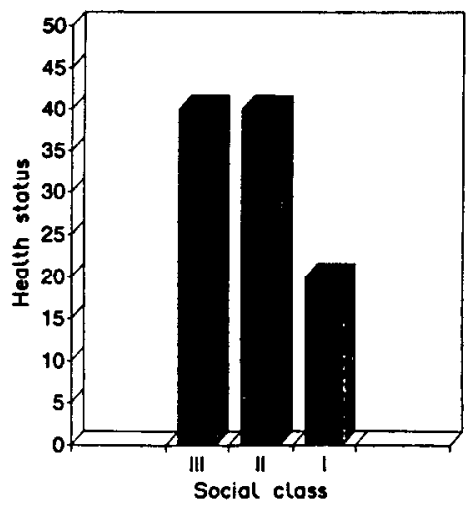

Fig. 4. Hypothetical distribution of health across social classes.

where $s_{j h}$ is the $j$ th group's share of the population's health and $s_{j p}$ is the $j$ th group's population share. The greater the difference between $s_{j h}$ and $s_{j p}$, the greater the degree of inequality. To calculate the degree of inequality one takes the absolute value of these differences, sums them up and divides by 2 .

Before considering the defects of ID as a measure of inequality in the present context, it is worth noting that ID is similar to the Information Theory (IT) approach to the measurement of inequality [13]. To see the similarity note that on individual-level data with $i=1, \ldots, \mathrm{n}$ individuals the ID would be

$$
\text { ID }=\frac{1}{2} \Sigma_{i}\left|s_{i h}-(1 / n)\right| .
$$

Thus under complete equality everyone's share of health would be equal to their population share. Compare this with the following variation on the IT theme which-like ID-implies an absolute distance concept [14]

$$
\text { IT }=\frac{1}{2} \Sigma_{i}\left[s_{i h}^{2}-(1 / n)^{2}\right] .
$$

The similarity to ID is obvious.

Given the similarities between ID and IT, it is clear that ID will have similar properties to IT. Though this would make it attractive in another context (e.g. the measurement of income inequality), it makes it singularly unattractive in the present context, even if the data are aggregated into socioeconomic groups. The defect is obvious. The ID suffers from the same shortcoming as the pseudo-Lorenz curve discussed

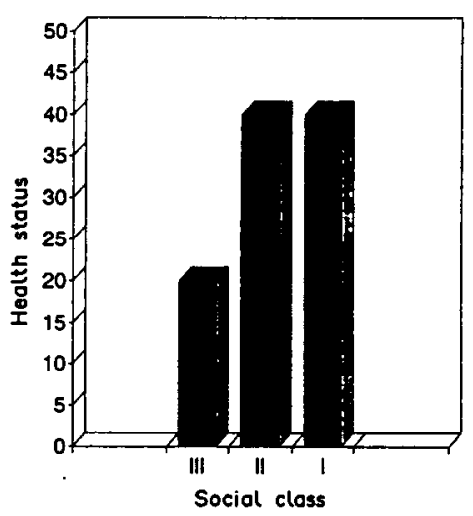

Fig. 5. Hypothetical distribution of health across social classes. 
above: it is insensitive to the socioeconomic dimension to inequalities in health. What matters in the ID is simply how each socioeconomic group's share of the population's health compares with its population share, not how this disparity compares with the socioeconomic group's socioeconomic status. The ID would, for example, register a positive value even when no class gradient exists. For example, the data in Fig. 2 give an ID value of 0.133 . Moreover, the ID would fail to detect a reversal in the class gradient as in Figs 4 and 5: indeed, in both cases the ID takes the same value as in Fig. 2.

Both of these difficulties emerge in Koskinen's [6] analysis of trends in cause-specific inequalities in mortality in England and Wales. In an attempt to overcome them Koskinen puts a \pm sign in front of the ID value if there is no clear class gradient and a minus (plus) sign if the gradient seems to favour the lower (upper) socioeconomic groups. Thus the ID for male mortality from leukemia is $\pm 1.6 \%$ in 1961 and $-1.0 \%$ in 1971 , whilst the ID value for male mortality from ischaemic heart disease is $-2.9 \%$ in 1951 and $+2.6 \%$ in 1961 . Clearly this introduces a substantial element of arbitrariness into the measurement exercise (when is a gradient not a gradient?) and a considerable amount of confusion into the interpretation of the results (how is $\pm 1.6 \%$ to be compared with $-1.0 \%$ ?).

\section{The slope and relative indices of inequality}

Unlike the Lorenz curve, the pseudo-Lorenz curve and the ID, the slope index of inequality $[4,5,15]$ and its relative difference counterpart-the relative index of inequality - do reflect the socioeconomic dimension to inequalities in health. The approach involves calculating the mean health status of each socioeconomic group and then ranking classes by their socioeconomic status (not by their health). The height of each bar in Fig. 6 represents the mean health status of the class in question and the width represents the fraction of the population in the class. The slope index of inequality (SII) is then defined as the slope of the regression line showing the relationship between a class's health status and its relative rank $\left(R_{j}\right)$ in the socioeconomic distribution. It can be interpreted as the absolute effect on health of moving from the lowest socioeconomic group through to the highest [4].

Because the data are grouped data, the error term of the regression equation is heteroskedastic [16, p. $323 ; 17$, p. 268]. Ordinary Least Squares (OLS) is therefore inefficient, although still unbiased. The appropriate estimator-and that used in empirical work to date-is Weighted Least Squares (WLS). The WLS estimate of the SII can be obtained by using the formula given on p. 324 of [16] or more simply [17, p. 268] by running OLS on the following transformed equation

$$
h_{j} \sqrt{ } n_{j}=\alpha \sqrt{ } n_{j}+\beta x_{j} \sqrt{ } n_{j}+u_{j}
$$

where $h_{j}$ is the health score of class $j, n_{j}$ is the size of class $j, x_{j}$ is the relative rank of persons in class $j$ and $u_{j}$ is a homoskedastic error term. Thus the appropriate weights in this case are the class sizes (or proportions): the health and relative rank of each class are then multiplied by the square root of the

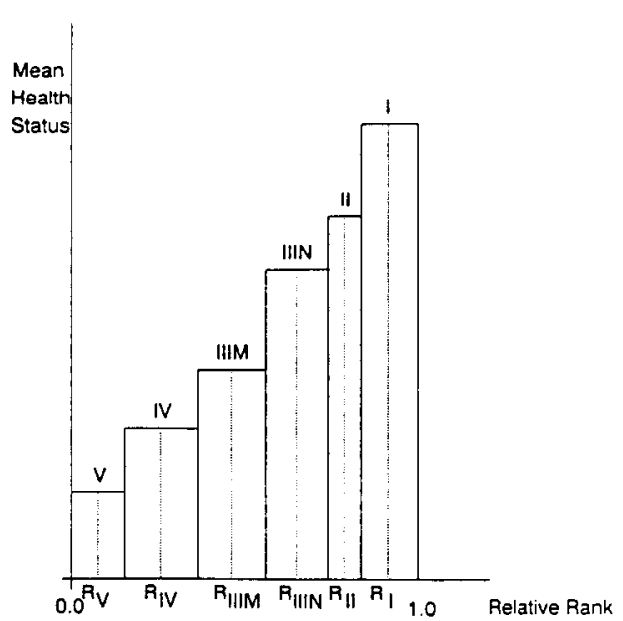

Fig. 6. Slope index of inequality.

appropriate weight. It is worth emphasizing that there is no constant term in the transformed equation: instead $\sqrt{ } n_{j}$ is entered as a regressor.

It is clear from Fig. 6 that the SII avoids the defects of the range measure: it reflects the experiences of the entire population and it is sensitive to the distribution of the population across socioeconomic groups. Moreover, because it ranks socioeconomic groups by socioeconomic status rather than by health, the SII reflects the socioeconomic dimension to inequalities in health. It may be verified, for example, that the SII for the data in Figs 2, 4 and 5 are $0.00,-30.00$ and +30.00 respectively - a set of results that accords with the notion of inequality as constituting a class gradient.

One additional noteworthy feature of the SII is its sensitivity to the mean health status of the population. Suppose that everyone's health doubled. Then the bars in Fig. 6 would become twice as high and the SII would double. Whether inequality has doubled is a moot point [18]: relative differences have remained the same, but absolute differences have widened. If it is the former that are regarded as important, the SII might be divided by the mean level of health, in which case a doubling of everyone's health would leave the resultant index unaffected. Pamuk [15] refers to this index as the relative index of inequality (RII). Clearly depending on changes in the mean vis-a-ais changes in the SII, the SII and RII may well move in opposite directions over time. This actually happens in Pamuk's [5] analysis of trends in inequality across social classes in England and Wales: the SII is smaller in 1949-53 than in 1930-32, whilst the opposite is true of the RII.

\section{The concentration index}

Another index that has been used in the analysis of inequalities in health is the concentration index [19]. As with the SII and RII, people are ranked not by their health but by their socioeconomic status, beginning with the most disadvantaged. The curve labelled $g^{\text {hlth }}(s)$ in Fig. 7 is a concentration curve for health. It plots the cumulative proportions of the population (beginning with the most disadvantaged and ending with the least disadvantaged) against the cumulative proportions of health. If health is equally distributed 


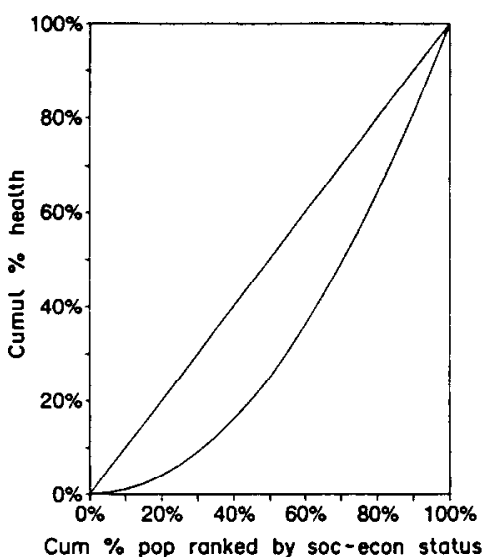

Fig. 7. Health concentration curve.

across socioeconomic groups, the concentration curve will coincide with the diagonal. If poor health is concentrated in the lower socioeconomic groups, the health concentration curve lies below the diagonal. The further $g^{\text {blth }}(s)$ lies from the diagonal, the greater the degree of inequality in health.

The health concentration index-denoted by $C$-is defined as twice the area between the concentration curve and the diagonal. This index provides a measure of the extent of inequalities in health that are systematically associated with socioeconomic status. Following the convention in the tax literature [20] the concentration index is defined as positive when the concentration curve lies below the diagonal and negative when it lies above the diagonal. Thus the lowest value that $C$ can take is -1 : this occurs when all the population's health is concentrated in the hands of the most disadvantaged person (so that $g^{\text {blth }}(s)$ is $\Gamma$-shaped). The maximum value the index can take is +1 : this occurs when all the population's health is concentrated in the hands of the least disadvantaged person (so that $g^{\text {hlth }}(s)$ is $ل$-shaped).

It is worth noting that concentration curve approach can also be used when it is inequality in ill-health that is being assessed. In this case the concentration curve lies above the diagonal if illness is concentrated amongst the lower socioeconomic groups. The illness concentration index-defined as twice the area between the concentration curve and the diagonal-is positive when the concentration curve lies below the diagonal (illness is concentrated amongst the higher socioeconomic groups) and negative when it lies above the diagonal (illness is concentrated amongst the lower socioeconomic groups).

That the concentration index avoids the defects of the range measure is clear: it reflects the experiences of the entire population and it is sensitive to the distribution of the population across socioeconomic groups. Moreover, because it ranks individuals by socioeconomic status rather than by health (hence the ' $s$ ' in brackets after $g^{\text {blh }}$ in Fig. 7 and the ' $h$ ' in brackets after $g^{\text {hth }}$ in Fig. 1), the concentration index also ensures that the socioeconomic dimension to inequalities in health is taken into account.

The concentration index will give the same result as the Lorenz curve (or the pseudo-Lorenz curve if grouped data are being used) only if the ranking of units of analysis by health is the same as the ranking by socioeconomic status [21]. This is illustrated in Fig. 8 which shows the concentration curves corresponding to the data in Figs 2, 4 and 5. Only the concentration curve corresponding to Fig. 5 has the same shape as the pseudo-Lorenz curve of Fig. 3. This is because this is the only example where the ranking of classes by health coincides with the ranking by socioeconomic status. The concentration curves corresponding to Figs 4 and 5 lie above and below the diagonal respectively, reflecting the fact that in Fig. 4 the class gradient favours the lowest class, whilst in Fig. 5 the gradient favours the top class. This is refiected in the fact that the concentration indices are the same in absolute value: the concentration indices corresponding to Figs 4 and 5 are -0.133 and +0.133 respectively. Finally, the concentration curve corresponding to Fig. 2 crosses the diagonal at the $50 \%$ point, resulting in a concentration index value of 0.000 . This result makes sense if one accepts the argument that there is no class gradient in Fig. 2 and accords with the zero value of the SII in this example. Figure 8 does, however, make explicit something that is implicit in the judgement that there is no inequality in Fig. 2, namely that the relative advantage of class III ought to be allowed to offset the relative disadvantage of class II. What this means in terms of Fig. 8 is that the area above the diagonal (which reflects the advantaged position of the lowest class) ought to be offset against the area below the diagonal (which reflects the disadvantaged position of the middle class and the advantaged position of the top class).

The result above concerning the importance of the rankings by health and by socioeconomic status is helpful when interpreting the two studies to date that have employed the pseudo-Lorenz curve approach $[4,10]$. In their analysis of trends in inequalities in health across social classes, Preston et al. report that "for each of the time periods under consideration mortality decreases monotonically from social class I to social class V" [4, p. 249]. In this case, therefore, the pseudo-Lorenz curve and the concentration curve approaches give the same result. But this is unlikely to be the case generally, especially as the number of socioeconomic groups used increases. Preston et al. for example, employ their pseudo-Lorenz curve to compare inequality across 182 occupational classes in

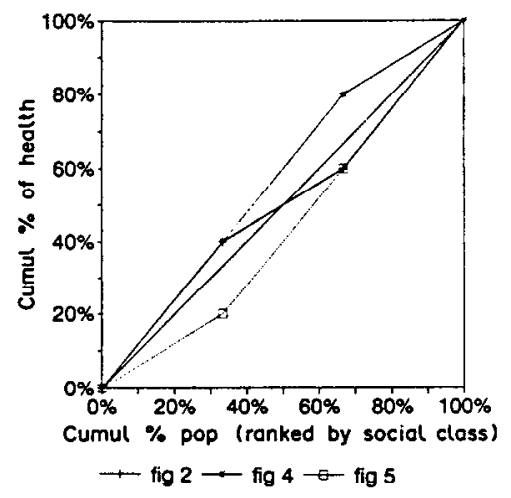

Fig. 8. Concentration curves for Figs 2, 4 and 5. 
1921-23 and 1970-72. Though they do not indicate how the rankings by mortality and by socioeconomic status compare, it would be most surprising if they were identical. But even with fewer socioeconomic groups, differences in health and socioeconomic status rankings emerge: Leclerc et al. [10], in their analysis of inequalities in France, rank by mortality and end up ranking foremen well ahead of managers. We illustrate the implications of the different rankings in Section 4.

\section{The $R I I$ and concentration index compared}

This completes our review of the inequality measures employed to date in the inequalities in health literature. Of the indices reviewed only the SII, RII and concentration index meet what would appear to be the minimal requirements of an inequality measure in this context: (i) that it reflect the socioeconomic dimension to inequalities in health; (ii) that it reflect the experiences of the entire population (rather than just, say, social classes I and V); and (iii) that it be sensitive to changes in the distribution of the population across socioeconomic groups.

Of these three indices, the SII differs from the other two in that it is sensitive to changes in mean health status. As we have noted above, this will make the SII attractive to those who wish to emphasize absolute differences between people or groups rather than relative differences. In this regard it is worth noting that it is possible to generalize the concentration curve so that it too is sensitive to changes in mean health status. This generalized concentration curve is illustrated in Fig. 9 and is the analogue of Shorrock's [22] generalized Lorenz curve. The curve in Fig. 9 is simply the standard concentration curve multiplied by the mean level of health (in this case assumed to be 10 units). It therefore shows the cumulative percent of the population (ranked, as before, by socioeconomic status) graphed against the cumulative amount of health (rather than the share of health). The generalized concentration index is then defined as twice the area between the generalized concentration curve and the diagonal.

In effect, then, there would appear to be just two measures of inequality that would seem suited to the measurement of inequalities in health: the SII and the generalized concentration index (if one wants an

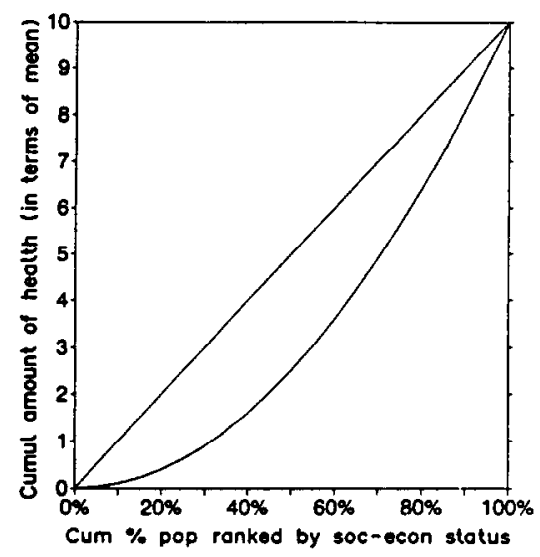

Fig. 9. Generalized concentration curve. index that is sensitive to changes in mean health status), and the RII and standard concentration index (if one does not). In fact, the choice is simpler than this, since the SII and the generalized concentration index are closely related, as are the RII and the standard concentration index. The latter can be shown [21] to be equal to

$$
C=2 \operatorname{cov}(x, h) / \mu \text {, }
$$

where $\operatorname{cov}(x, h)$ is the covariance between the relative rank $x$ and health $h$, and $\mu$ is the mean level of health. The similarity with the SII becomes apparent when one notes that $\operatorname{cov}(x, h)$ can be found by running a regression of $h$ on $x$ [23]. The slope coefficient, $\beta$, from this regression is equal to

$$
\beta=\operatorname{cov}(x, h) / \operatorname{var}(x),
$$

where $\operatorname{var}(x)$ is the variance of the relative rank variable. Hence

$$
C=2 \operatorname{var}(x)(\beta / \mu) .
$$

It may be verified that this result holds irrespective of whether individual-level data or grouped data are used: calculation of $C$ on grouped data by the more common method of linear approximation [24] gives precisely the same result as is obtained by substituting the WLS estimate of $\beta$ into the formula for $C$ above.

It thus emerges that the RII $(\beta / \mu)$ is equal to the concentration index divided by twice the variance of the relative rank variable. Moreover, since the generalized concentration index is the standard concentration index multiplied by mean health, the SII is equal to the generalized concentration index divided by the variance of the relative rank variable. This result is useful, since it shows that there is little to choose between the RII (or SII) and the concentration index (or generalized concentration index). However, although the RII and SII have perhaps a more immediate graphical interpretation than a concentration curve, the latter is arguably more useful as a visual device when performing comparisons across countries or over time. This is especially true when grouped data are used and the sizes of the groups are not constant. In this case comparing a Fig. 6 drawn for one time period with that drawn for another becomes difficult. The same is not true of the concentration curve where, as will be seen below, before-and-after and them-and-us comparisons are both straightforward and revealing.

\section{INEQUALITIES IN MORBIDITY}

Ever since the publication of the Black Report [1] there has been a good deal of interest in comparative studies of inequalities in morbidity. Have inequalities in morbidity in country $X$ been increasing or diminishing over time? Are they more pronounced than the inequalities in morbidity in country $Y$ ?

There are those [25] who warn against such comparisons on the grounds that the variables concerned-morbidity and socioeconomic statusmay be interpreted differently in different countries and at different times. This is undoubtedly true. It may well be, for example, that the healih threshold below which people define themselves to be in poor health changes over time or varies across countries. 
But whilst this has clear implications for the mean morbidity level, it is not obvious that it has any implications for inequality in morbidity. This will only be affected if the threshold gradient changes over time or varies across countries. Have, for example, those in social class I changed their threshold more quickly (or slowly) than the rest of the population? Clearly, changes in the average threshold tell us nothing about any changes in the threshold gradient: such changes may or may not occur when the mean threshold changes; indeed, such changes may occur even when the mean threshold is not changing. What the argument does imply is that in comparing inequalities in morbidity over time or across countries it is probably better to employ a measure of inequality that is independent of the mean level of morbidity.

In the several recent comparative studies of inequality authors have tended to rely on the range measure of inequality or on a visual inspection of the class gradient. In this section we show how the data reported in some of these studies can usefully be reworked to obtain a more precise picture of inequality. Given our comments above, we use a measure of inequality that is independent of the mean level of morbidity. Given the visual advantage of the concentration curve over the RII, we have employed the former in what follows. Since the concentration index and the RII are related, it is clear that similar conclusions would have been reached if the RII had been used instead.

\section{Inequalities in morbidity: Britain and Sweden compared}

Lundberg [26] and Vagerö and Lundberg [27] report the results of a comparison of inequalities in morbidity across social classes in Sweden and Britain. In contrast to other cross-country comparisons of social class inequalities in morbidity [25], these studies employ the British Registrar General's social class classification system for both countries. Table II of their paper contains information on the proportion of each country's population falling into social classes I, II, IIN, IIIM, IV and V, as well as the relevant age-standardized rates of prevalence of long-standing illness for men and women aged 16 and over. Though the relative prevalence figures are normalized to form

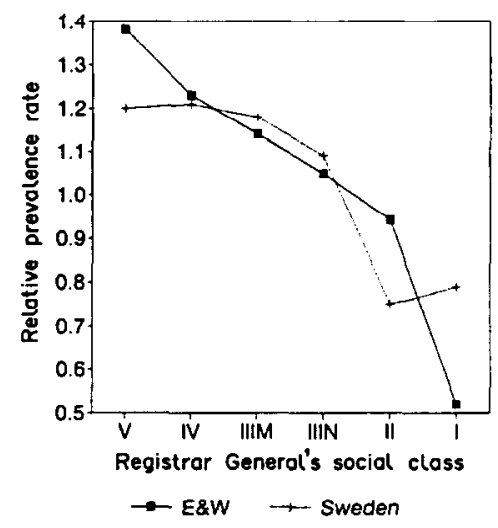

Fig. 10. Prevalence of chronic illness, England and Wales, and Sweden, 1981

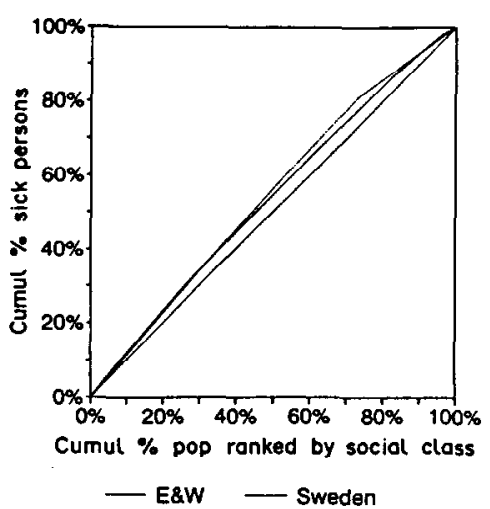

Fig. 11. Inequalities in morbidity, England and Wales, and Sweden.

a product of 1.00 , the normalization factor cancels out when the $j$ th class's share of the sick population is calculated. Thus the latter can be found by multiplying each class's prevalence rate by its population share and dividing the sum of these products into the product for the $j$ th class.

The variation of prevalence rates across social classes can be seen from Fig. 10. The range is evidently greater in the case of Britain: the ratio of class V's prevalence rate to that of class $I$ is 2.65 in Britain, but only 1.52 in Sweden. The concentration curves, however, tell a quite different story: see Fig. 11. The Swedish curve cuts the British curve from below and is, on average, further from the diagonal than the British curve. This reflects the fact that whilst in Britain the class gradient declines fairly gradually, in Sweden there is relatively little inequality amongst the lower social classes but a substantial gap between the top two classes (which together account for almost $30 \%$ of the Swedish population) and the rest of the population. This feature of the Swedish morbidity distribution is, of course, not picked up by the range measure. Not surprisingly, in view of the remarks above, the concentration index for Sweden is larger in absolute value than that for Britain $(-0.087$ compared to -0.071$)$.

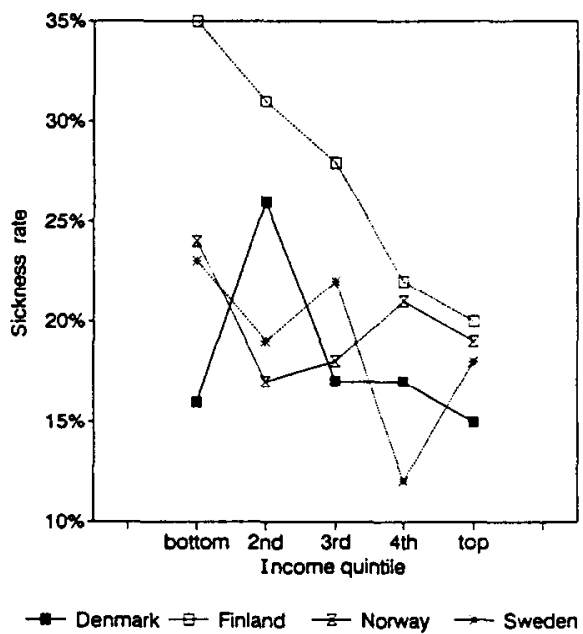

Fig. 12. Prevalence of chronic illness in the Nordic countries. 
Inequalities in morbidity: the Nordic countries compared

Figure 12, based on Table 6 of Ref. [7], shows the variations across income groups in age-standardized chronic sickness rates of persons aged 15-64 in Denmark, Finland and Sweden in 1972.

The illness concentration curves corresponding to these data are shown in Fig. 13. It is apparent that the concentration curves for Finland and Sweden lie everywhere above the diagonal, whilst the curves for Denmark and Norway cross the diagonal. The Danish curve crosses from below, reflecting the fact that-according to the data reported in Ref. [7]those in the bottom income quintile in Denmark appear to be less likely than anyone else in Denmark (apart from those in the top income quintile) to have a chronic health problem. The Norwegian concentration curve, by contrast, crosses the diagonal twice, first from above, reflecting the fact that persons in the fourth income quintile in Norway are apparently more prone to chronic sickness than anybody else except those in the bottom quintile, and then from below, reflecting the low illness rate of the top quintile. It is also apparent that the Finnish concentration curve lies everywhere above the Swedish curve, which in turn lies everywhere above the Norwegian curve. This implies that inequalities in health-as measured by chronic sickness-are unambiguously less pronounced in Sweden than in Finland, but more pronounced in Sweden than in Norway. Thus not only does Finland have the highest average chronic sickness rate of the Nordic countries; it also appears to have the highest degree of inequality.

Some idea of the extent of inequality in these countries can be gleaned from the concentration index values: Denmark: -0.048 ; Finland: -0.115 ; Norway: -0.024 ; Sweden: -0.072 . The ranking of the last three confirms our earlier remarks about the concentration curves. Interestingly, despite the low sickness rate amongst the bottom quintile in Denmark, the overall picture is one of inequality favouring the rich. Indeed, on balance, there isaccording to the concentration index scores-more inequality in health in Denmark than in Norway. Interestingly, the opposite conclusion is reached if one uses the range measure: the ratio between the lowest and highest quintile is 1.06 in Denmark and 1.26 in Norway.

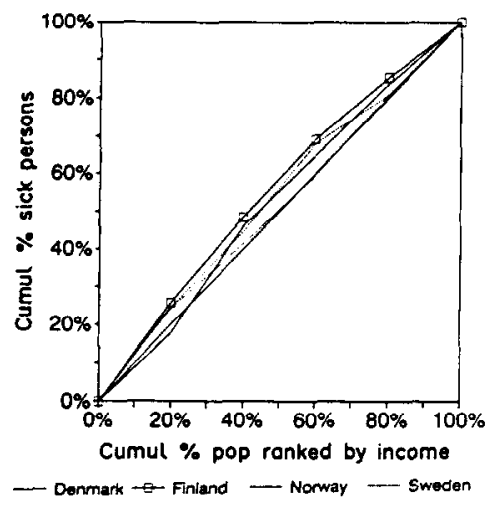

Fig. 13. Inequalities in morbidity in the Nordic countries.

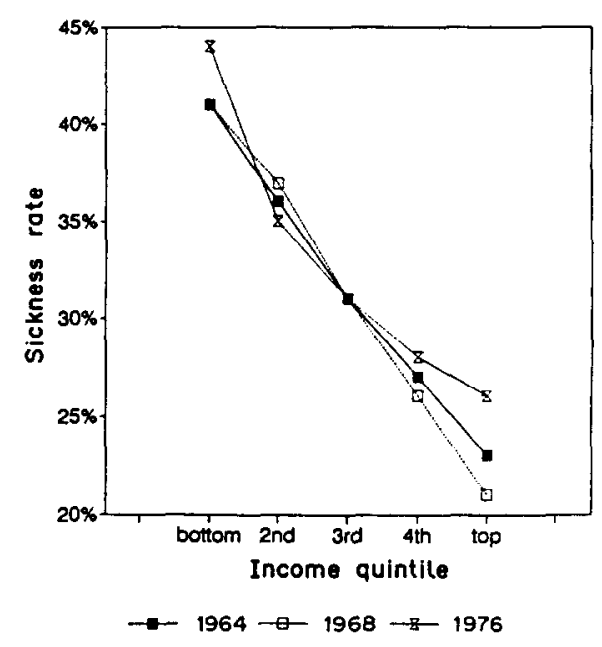

Fig. 14. Prevalence of chronic illness, Finland 1964-76.

\section{Trends in inequality in morbidity in Finland}

Figure 14, based on Table 3 of Ref. [7], shows the age-standardized chronic illness rate amongst persons aged $15+$ for each quintile of family income in Finland in 1964, 1968 and 1976.

The illness concentration curves corresponding to these data are shown in Fig. 15. It is evident that all three illness concentration curves lie everywhere above the diagonal. Inequalities in health thus existed in each year and unambiguously favoured the better off. It is also evident that the 1968 concentration curve lies everywhere above the 1964 curve, implying that inequalities in health were unambiguously greater in 1968 than in 1964. Between 1968 and 1976 the illness concentration curve moves out at the bottom of the income distribution but inwards at the top, so that curves for the two years cross. The outwards movement at the bottom reflects the deterioration in health of the bottom quintile. It is also evident that the curves for 1964 and 1976 also cross.

Some idea of the extent of inequality in the three years can be got from the concentration index values: -0.114 in $1964,-0.131$ in 1968 and -0.105 in 1976 . The fact that the index value for 1964 is lower in absolute value than the index score for 1968 is to be expected given the relative positions of the

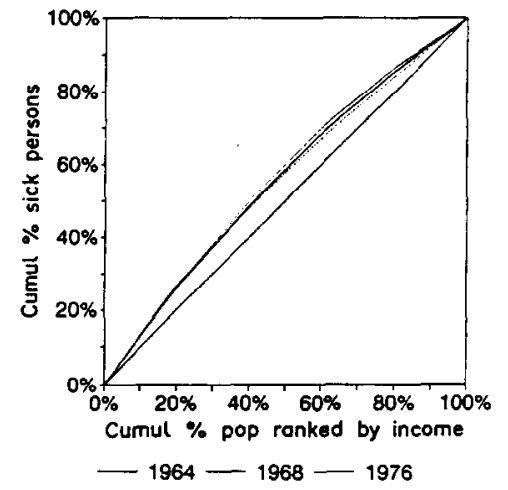

Fig. 15. Inequalities in morbidity, Finland 1964-76. 
concentration curves for these two years. That the index score for 1976 is lower than that for 1968 indicates that on balance inequalities in health were less pronounced in 1976 than in 1968. Indeed, the index scores indicate that on balance there was less inequality in health in Finland in 1976 than in 1964. It is worth noting that in this case the range measure leads to the same conclusion.

\section{Choice of morbidity indicator and the measurement of inequality}

There is another type of comparison where measures of inequality are useful, namely in comparison across health measures. Blaxter [25], for example, finds that class gradients tend to be steeper when morbidity is measured in terms of chronic illness than when measured in terms of restricted activity days. Unfortunately, Blaxter's data cannot be reworked into concentration curves, since the data are not presented in tables; nor is there any indication of the distribution of the population across socioeconomic groups. Our data in this section are therefore primary data. They relate to Italy and the Netherlands and are taken from the 1985 Italian Health Care Consumption Survey [28] and the combined 1981 and 1982 Dutch CBS Health Interview Survey.

We explore the implications of choosing between three measures of morbidity: (i) chronic sickness (did the person suffer from a chronic condition?), (ii) restricted activity days (we have examined simply the percentages reporting some restricted activity days), and (iii) self-perceived health (we have distinguished simply between people who regarded their health as at least good and those who regarded it as less than good). Our chronic illness indicator fits in with Blaxter's [25] medical model, in which ill-health is defined in terms of a deviation from physiological norms. Our restricted activity days (RADs) indicator, by contrast, fits in with her social-interactional or functional model, in which ill-health is defined in terms of a lack of ability to perform 'normal' tasks or roles. Finally, our self-assessed health indicator fits in with Blaxter's subjective model, in which ill-health is defined in terms of the individual's perception.

Figures 16 and 17 show the percentage of persons in each income group reporting chronic sickness, 'not

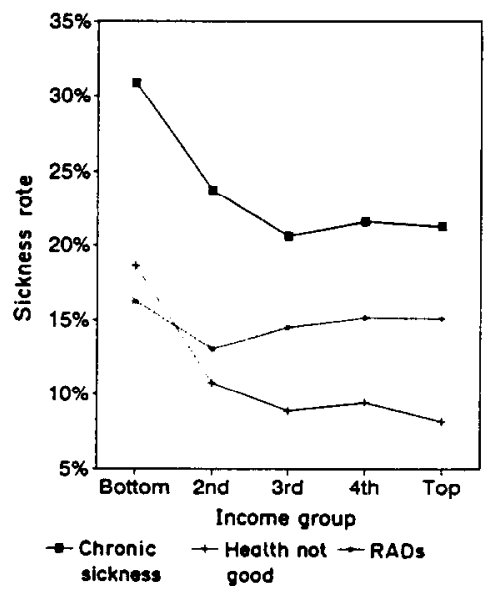

Fig. 16. Sickness rates in Italy.

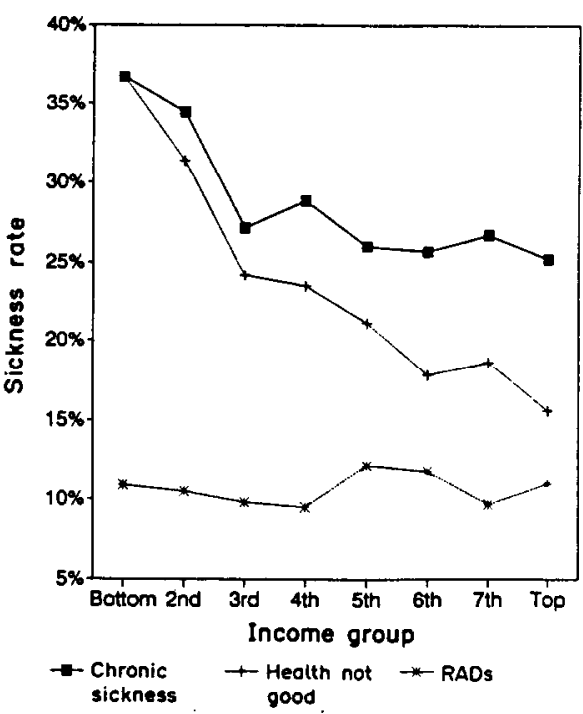

Fig. 17. Sickness rates in The Netherlands.

good' health, and at least one RAD. The fact that the proportion of the sample reporting their health to be 'not good' is smaller in Italy than in the Netherlands probably stems at least in part from the fact that the phrasing of the question was different in the two countries, though, of course, threshold effects may also be at work. Of more interest in the present context is the fact that there are clear gradients in both countries for the first and second health indicators, but not for the RADs indicator. This is consistent with Blaxter's [25] results: she found class gradients in most countries in chronic illness and self-perceived health, but detected very little variation across occupational groups in the proportions of persons reporting restricted activity days.

By combining the data in Figs 16 and 17 with information on the distribution of the sample across the income classes, it is possible to plot the concentration curves in Figs 18 and 19. In both countries the same picture emerges: the concentration curve lies everywhere above the diagonal for the chronic sickness and health-not-good indicators but crosses the diagonal in the case of the RADs indicator. According to the first two indicators, therefore, inequalities in morbidity exist in both countries and favour the better-off. The index values in Table 1 show that on balance inequality based on the RADs indicator favours the better-off in Italy but the worse-off in the Netherlands. The index values also indicate that, irrespective of which morbidity measure one chooses, inequalities in health across income groups appear to be slightly larger in Italy than in the Netherlands.

More interesting in the present context is the finding that in both countries it is the RADs that give rise to the smallest amount of inequality, the chronic illness indicator that produces the next smallest amount, and self-perceived health which produces the biggest amount. Indeed, the differences in the index values for the chronic illness and self-perceived health indicators are remarkably similar: in both countries the index for the latter is a little over twice that of the former. Inequalities in health thus do seem to be fairly pronounced when ill-health is measured by chronic 


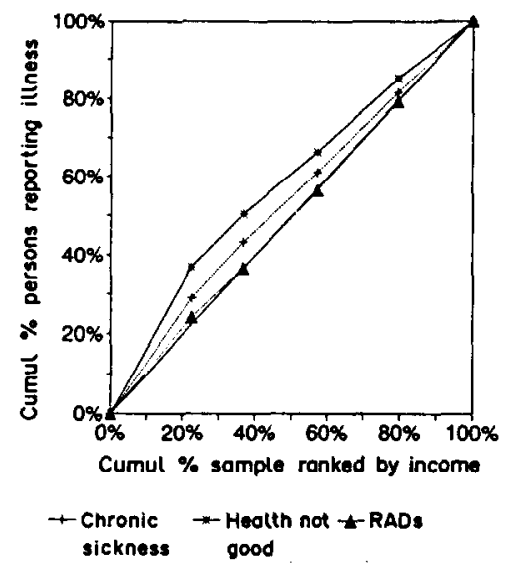

Fig. 18. Inequalities in morbidity, Italy 1985.

sickness, as Blaxter notes. But they are even more pronounced when one uses self-perceived health as the morbidity indicator.

\section{INEQUALITIES IN MORTALITY}

As with inequalities in morbidity the Black Report [1] did much to stimulate interest in comparative studies of inequalities in mortality. In Britain, for example, as indicated in the Introduction, there has been a good deal of debate about whether inequalities in mortality have been increasing or diminishing. But there has also been a growing interest in crosscountry comparisons [29]. Some of the recent literature in this area has employed the RII, whichas noted above-would appear to be a reliable measure of inequality in this context. Other work, however, has been based on less reliable measures, such as the range, the pseudo-Lorenz curve and the ID. In this section we show how the data in some of these studies can be reworked to obtain the more reliable concentration index measure of inequality.

Inequalities in death: England and Wales, and Sweden compared

Vagerö and Lundberg [27] report the results of a comparison of inequalities in mortality across social class in Sweden and England and Wales. As in their

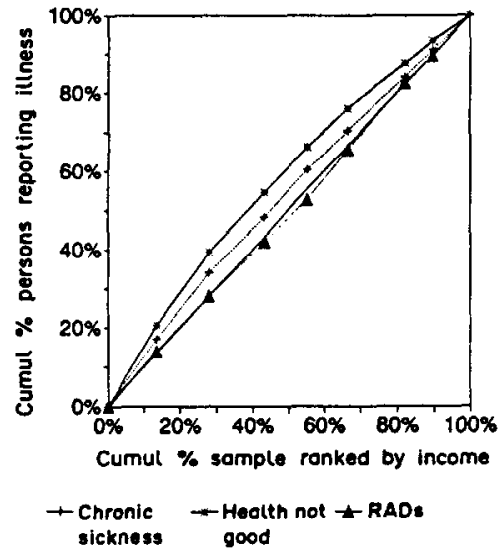

Fig. 19. Inequalities in morbidity, The Netherlands 1981-82.
Table 1. Concentration indices for Italy and Netherlands

\begin{tabular}{lcc}
\hline & Italy & Netherlands \\
\hline RADs & -0.005 & 0.011 \\
Health not good & -0.167 & -0.154 \\
Chronic illness & -0.075 & -0.071 \\
\hline
\end{tabular}

comparative study of morbidity inequalities reported above, the authors employ the British Registrar General's social class classification system for both countries. Table I of their paper contains information on the proportion of each country's population falling into social classes I + II, IIN, IIIM, and $\mathrm{IV}+\mathrm{V}$, as well as the relevant age-standardized death rates for men aged $20-64$.

With this information it is possible to plot concentration curves for the two countries as in Fig. 20. It is evident that the Swedish concentration curve lies everywhere below that of England and Wales, implying that inequalities in mortality are unambiguously higher in England and Wales than in Sweden. This is borne out by the concentration indices for the two countries, which are -0.081 and -0.042 respectively. The ranking of the two countries is in this case the same as that obtained using the range measure of inequality. It is interesting to note, however, that the percentage difference between the concentration index values $(94.5 \%)$ is considerably greater than the percentage difference in the ratio of class IV $+V$ death rates to class I + II death rates (16.5\%).

Trends in inequalities in death: a comparison of Finland, and England and Wales

Leclerc et al. [10] report the results of a comparative study of inequalities in mortality in three countries using the pseudo-Lorenz curve approach. Their analysis provides a useful illustration of the advantages of the concentration curve approach over the pseudo-Lorenz curve approach and the dangers of using the latter.

Table 3 of Ref. [10] indicates the distribution across classes of the population aged 35-44 in England and Wales, as well as the number of deaths occurring in this age-band in each social class. Table 4 of Ref. [10] indicates similar information for 8 socioeconomic

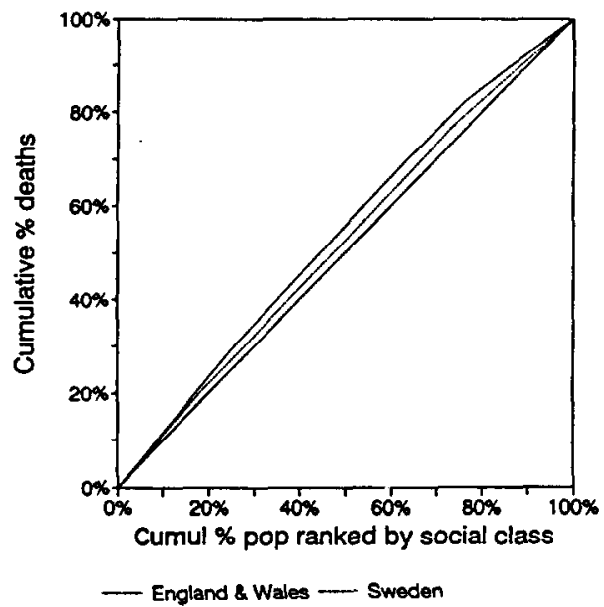

Fig. 20. Inequalities in mortality, England and Wales, and Sweden. 
groups for Finland. In both countries there are substantial differences in the ranking of socioeconomic groups by mortality rate and by socioeconomic status. In England and Wales, for example, classes IV and IIIN both have higher mortality rates than class V, whilst in Finland the socioeconomic groups with the third and fourth highest mortality rates are socioeconomic groups 3 and 1 (the latter being the group with the highest status).

Figure 21 shows the concentration curves for Finland and England and Wales. The Finnish curve is extremely irregular; indeed, it crosses the diagonal and the curve for Enland and Wales. Overall, however, it is evident that it is England and Wales that has the higher degree of inequality. This is borne out by the concentration indices for the two countries $(-0.074$ for Finland and -0.082 for England and Wales). Interestingly, this conclusion is precisely the opposite of that reached by Leclerc et al:: their pseudo-Gini coefficient for Finland was larger in absolute value than that for England and Wales.

Trends in inequalities in potential life lost in England and Wales

Comparisons such as those in Figs 20 and 21 take no account of the age at which people die [2]. That the gap between classes in the risk of dying before the age of 64 is greater in England and Wales than in Sweden does not mean that the gap between classes in the age at death of those dying prematurely is greater in England and Wales than in Sweden. A more attractive measure of mortality that takes into account not only the risk of premature death but also the age at which premature death occurs (if it does) is the number of years of potential life lost [30].

Blane et al. [31] report the results of an analysis of social class inequalities in potential years of life lost in England and Wales. Their Table III reports inter alia social classes differences in the annual age-standardized rate of potential years of life lost for men aged 15-64 for the years 1971 and 1981 .

By combining these rates with information on the populations in these years [32-35] it is possible to plot a concentration curve showing the cumulative percentage of the population (ranked by social class)

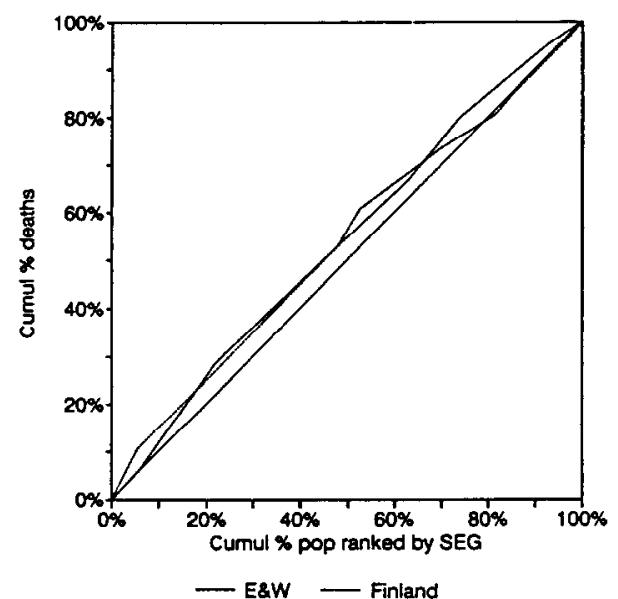

Fig. 21. Inequalities in mortality, England and Wales, and Finland.

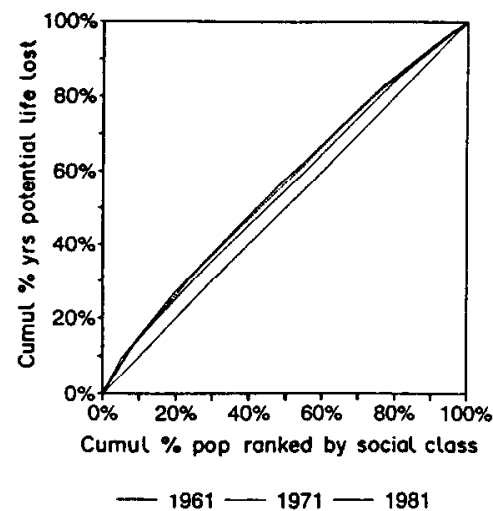

Fig. 22. Inequality in potential life lost.

against the cumulative percentage of years of potential life lost. Such curves are shown in Fig. 22 for 1961, 1971 and 1981: the age-standardized rates of potential years of life lost for 1961 were calculated by us from Ref. [36]. It is evident that the 1971 curve crosses the 1961 from below: this reflects the improvement in the relative position of class $V$ but the deterioration in the relative position of classes IV and $V$ combined. In this case, therefore, the comparison of inequality in the two years is ambiguous, though for the most part the 1971 curve lies above the 1961 curve. By contrast the comparison between 1971 and 1981 is unequivocal: the 1981 curve lies everywhere above the 1971 curve, implying that inequalities in potential life lost unambiguously increased during the 1970s. The comparison between 1961 and 1981 is also unambiguous: the 1981 curve lies everywhere above that of 1961.

The indices for the years 1961, 1971 and 1981 respectively are: $-0.083,-0.099$ and -0.118 . The latter two figures come as no surprise given the concentration curves for 1971 and 1981. That the 1971 figure is greater in absolute value than the 1961 figure reflects the fact that the area between the two curves prior to the crossover is much smaller than the area after the crossover. Interestingly, the range measure of inequality as used by Blane et al. (the ratio of the two extreme classes' rates) tells the opposite story for the 1961/1971 comparison (the ratios are 2.11 for 1961 and 2.10 for 1971) and a rather more dramatic story for the 1971/1981 comparison (the V:I ratio jumps by $33 \%$, whilst the concentration index rises by a more modest $20 \%$ ).

\section{Choice of mortality indicator and the measurement of} inequality

The use of years of potential life lost on the $y$-axis of the concentration curve diagram rather than the number of deaths raises the question: which measure gives rise to the greater degree of inequality?

Table 2 shows the class distribution in England and Wales in 1981 of years of potential life lost and deaths. Both are age-standardized using the direct standardization method. The distribution of the population across social classes is taken from fiche $\mathrm{C} 03$ of Ref. [37]. The age-standardized rates of years of potential life lost and age-standardized death rates are taken from Table II of Ref. [31] and fiche D02 of 
Table 2. Distribution of years of potential life lost and deaths. Male population aged 20-64, England and Wales 1981

\begin{tabular}{lccc}
\hline $\begin{array}{c}\text { Social } \\
\text { class }\end{array}$ & $\begin{array}{c}\text { Male pop. aged } \\
20-64 \\
(\%)\end{array}$ & $\begin{array}{c}\text { Years of potential } \\
\text { life lost } \\
(\%)\end{array}$ & $\begin{array}{c}\text { Deaths } \\
(\%)\end{array}$ \\
\hline V & 6.0 & 11.1 & 9.8 \\
IV & 16.6 & 19.5 & 19.3 \\
IIIM & 36.0 & 37.2 & 37.9 \\
IIIN & 11.7 & 10.9 & 11.0 \\
II & 23.7 & 17.5 & 18.0 \\
I & 6.0 & 3.9 & 4.1 \\
\hline
\end{tabular}

Ref. [37] respectively. Figure 23 shows the corresponding concentration curves. It is apparent that the concentration curve for years of potential life lost lies everywhere outside that for deaths, implying that inequality is unambiguously greater if mortality is measured by years of potential life lost rather than by deaths. The implication is that those in the lower social classes are not only more likely to die prematurely, but also tend to die at an earlier age if they do die prematurely. This conclusion is, in fact, the same as that reached by Blane et al. [31] on the basis of a comparison of the class $\mathrm{V}: \mathrm{I}$ ratios of years of potential life lost and SMRs.

\section{CONCLUSIONS}

Our primary objectives in this paper were to provide a critical review of the various measures of inequality that have been employed in the literature on inequalities in health to date and to identify which of these measures are best suited to measuring health inequality. We identified six measures of inequality that have been used: the range; the Gini coefficient (and the associated Lorenz curve) [2, 3, 8, 9]; a pseudo Gini coefficient (and an associated pseudo Lorenz curve) $[4,10]$; the index of dissimilarity; the slope index of inequality; and the concentration index (and the associated concentration curve).

Only the slope index of inequality and the concentration index meet what we argued were the minimal requirements of an inequality measure in this context: that it reflect the socioeconomic dimension to inequalities in health; that it reflect the experiences of the entire population (rather than just, say, social classes $I$ and $V$ ); and that it be sensitive to changes in the distribution of the population across socioeconomic groups. The other four measures fail to satisfy

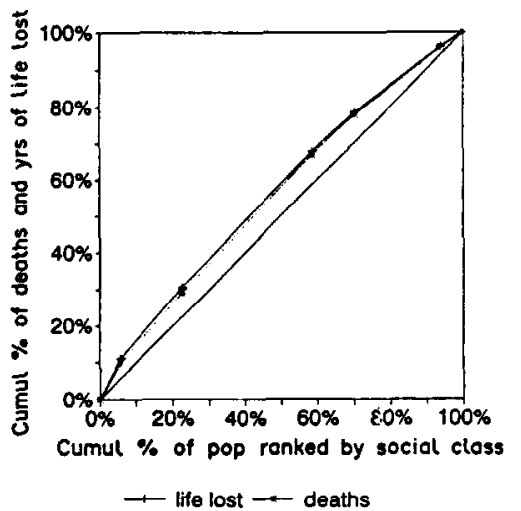

Fig. 23. Inequality in potential life lost and death. one or more of these criteria: the range measure fails to satisfy the second and third; the Gini coefficient, the pseudo-Gini coefficient and the index of dissimilarity all fail to satisfy the first. We also showed that the two reliable measures were related to one another: the relative version of the slope index of inequality, which emphasizes relative rather than absolute difference, turns out to be equal to the concentration index multiplied by twice the variance of the relative rank. It was also shown that the pseudo-Gini coefficient only gives the same result as these two inequality measures when the ranking of socioeconomic groups by health and by socioeconomic status coincide.

Our conclusions suggest, therefore, that if the aim of the exercise is to establish the extent of socioeconomic inequalities in health, one ought to interpret with caution the findings of studies that have employed one or other of the inequality measures that we have argued to be unreliable. By contrast our conclusions provide strong methodological support for the recent work of Pamuk [5, 15].

Our second objective was to illustrate the importance of having a reliable measure of inequality in comparative studies of inequalities in health. For the most part the results reported in the paper simply involve reworking results published elsewhere where authors had relied on an unreliable measure of inequality. In several cases we found that use of the concentration index (and by implication the relative index of inequality) leads to quite different conclusions concerning the extent of inequalities in health. Use of the range measure, for example, leads to the conclusion that there is less class-related inequality in chronic sickness in Sweden than in England and Wales, whilst use of the concentration index leads to precisely the opposite conclusion. Likewise, use of the pseudo-Lorenz curve leads to the conclusion that there is less inequality in death amongst the 35-44 age group in Finland than in England and Wales, whilst use of the concentration curve leads one to conclude the opposite.

It is, of course, not always the case that different inequality measures lead to different conclusions. We found, for example, that irrespective of whether one uses the range measure of inequality or the concentration index, inequalities in mortality in England and Wales in 1981 were more pronounced if mortality is measured by years of potential life lost than if measured simply by deaths. However, given the potential for misleading results and the ease with which the relative index of inequality and the concentration index can be computed, reporting one or the other in future comparative studies seems highly desirable. The same is true of the concentration curve: it is easy to graph and greatly facilitates comparisons of inequality.

Acknowledgements - We are grateful to the EC for financial support and to Frans Rutten--the project leader-for encouragement and advice. We are also grateful to the Centro Europa Ricerche (CER) in Rome and in particular to Stefania Gabriele for providing data from CER's Health Care Consumption Survey, and to Jan van Emmerick for help in analysing data from the Dutch Health Interview Survey. We are also grateful to Julian Le Grand and Richard Wilkinson for helpful comments on an earlier version of this paper. 


\section{REFERENCES}

1. Townsend P. and Davidson N. Inequalities in Health The Black Report. Penguin, Harmondsworth, 1982.

2. Illsley R. and Le Grand J. The measurement of inequality in health. In Health and Economics (Edited by Williams A.). Macmillan, London, 1987.

3. Le Grand J. and Rabin M. Trends in British health inequality: 1931-83. In Public and Private Health Services (Edited by Culyer A. J. and Jönsson B.) Blackwell, Oxford, 1986.

4. Preston S. H., Haines M. R. and Pamuk E. Effects of industrialization and urbanization on mortality in developed countries. In Solicited Papers Vol 2, IUSSP Ith International Population Conference, Manila. IUSSP, Liege, 1981.

5. Pamuk E. Social class inequality in mortality from 1921-1972 in England and Wales. Population Studies $39,17-31,1985$.

6. Koskinen S. Time trends in cause-specific mortality by occupational class in England and Wales. Unpublished paper presented at IUSSP 20th General Conference, Florence, 1985.

7. Lahelma E. and Valkonen T. Health and social inequities in Finland and elsewhere. Soc. Sci. Med. 31, 257-265, 1990

8. Le Grand J. Inequalities in health: The human capital approach. Welfare State Programme Discussion Paper 1, London School of Economics, 1985.

9. Le Grand J. An international comparison of distributions of ages-at-death. In Health Inequality in European Countries (Edited by Fox J.). Gower, Aldershot, 1989.

10. Leclerc A.. Lert F. and Fabien C. Differential mortality: some comparisons between England and Wales, Finland and France, based on inequality measures. Int J. Epidemiol. 19, 1001-1010, 1990.

11. Wilkinson R. G. Socioeconomic differences in mortality: interpreting the data on their size and trends. In Class and Health: Research and Longitudinal Dato (Edited by Wilkinson R. G.). Tavistock, London, 1986.

12. Marmot M. G., Adelstein A. M., Robinson N. and Rose $\mathrm{G}$. The changing social class distribution of class disease. Br. Med. J. ii, 1109-1112, 1978.

13. Theil H. Economics and Information Theory. North Holland, Amsterdam, 1967.

14. Cowell F. Measuring Inequality. Phillip Allan, Oxford, 1977.

15. Pamuk E. Social-class inequality in infant mortality in England and Wales from 1921 to 1980. Eur. J. Popul. 4, 1-21, 1988.

16. Kmenta J. Elements of Econometrics. Macmillan, New York, 1971.
17. Madalla G. S. Exonometrics. McGraw-Hill, London, 1979.

18. Sen A. On Economic Inequality. Clarendon, Oxford, 1973.

19. Wagstaff A., van Doorslaer E. and Paci P. Equity in the finance and delivery of health care: some tentative cross-country comparisons. Oxford Ret. Economic Policy 5, 89-112, 1989.

20. Lambert P. J. Tax progressivity: a survey of the literature. Working Paper 56, London, Institute for Fiscal Studies, 1985.

21. Jenkins $\mathbf{S}$. Calculating income distribution indices from microdata. National Tax J. 61, 139-142, 1986.

22. Shorrocks A. F. Ranking income distributions. Economica 50, 3-17, 1983.

23. Lerman R. I. and Yitzhaki S. A note on the calculation and interpretation of the Gini index. Economics Lell. 15, 363-368, 1984.

24. Fuller M. F. and Lury D. A. Statistics Workbook for Social Science Students. Philip Allan, Oxford, 1977.

25. Blaxter $M$. A comparison of measures of inequality in morbidity. In Health Inequality in European Countries (Edited by Fox J.). Gower, Aldershot, 1989.

26. Lundberg $O$. Class and health: comparing Britain and Sweden. Soc. Sci. Med. 23, 511-517, 1986.

27. Vagerö $D$. and Lundberg $O$. Health inequalities in Britain and Sweden. The Lancet ii, 35-36, 1989.

28. Bariletti A., Gabriele S., Marè $M$. and Piacentino D. Indagine sui consumi sanitarie delle familglie Italiane. Research Report, Centro Europa Ricerche, Rome, 1986.

29. Fox J. (Ed.) Health Inequality in European Countries. Gower, Aldershot, 1989.

30. Haenszel W. A standardized rate for mortality defined in units of lost years of life. Am. J. Publ. Hlth 40, 17-26. 1950.

31. Blane D., Davey Smith G. and Bartley M. Social class differences in years of potential life lost: size, trends and principal causes. Br. Med. J. 301, 429-432, 1990.

32. General Register Office. Census 1961: England and Wales Occupation Tables. HMSO, London, 1966.

33. OPCS. Census 1971: Great Britain Economic Activity Part IV. HMSO, London, 1975.

34. OPCS. Census 198I Great Britain: Economic Activity. HMSO, London, 1984.

35. Registrar General for Scotland. Census 1981 Scotland: Economic Activity. HMSO, Edinburgh, 1984.

36. OPCS. Registrar General's Decennial Supplement. England and Wales, 1961: Occupational Mortality Tables. HMSO, London, 1971.

37. OPCS. Occupational Mortality Decennial Supplement, 1979-80, 1982-83, Great Britain, Series DS No. 6. HMSO, London, 1986. 